\title{
Dynamic Optimization of Bus Line Schedule in Commuter Corridor Based on Bus IC Card Data
}

\author{
Zhihong Li $\mathbb{D}^{1},{ }^{1}$ Han Xu, ${ }^{1}$ Shiyao Qiu, ${ }^{1}$ Jun Liu, ${ }^{2}$ Kairan Yang, ${ }^{1}$ and Jiahao Wu ${ }^{1}$ \\ ${ }^{1}$ General Aviation Technology Beijing Laboratory, Beijing University of Civil Engineering and Architecture, \\ Beijing 100044, China \\ ${ }^{2}$ Institute of Electronic Studies, China Academy of Railway Sciences Group Co., Ltd, Beijing 100044, China \\ Correspondence should be addressed to Zhihong Li; lizhihong@bucea.edu.cn
}

Received 8 November 2021; Accepted 15 December 2021; Published 13 January 2022

Academic Editor: Yu-Sheng Ci

Copyright (C) 2022 Zhihong Li et al. This is an open access article distributed under the Creative Commons Attribution License, which permits unrestricted use, distribution, and reproduction in any medium, provided the original work is properly cited.

\begin{abstract}
The aim of this study was to explore the bus operating state of the city bus passenger corridor, taking the minimum bus operating cost and passenger travel cost as the objective function, taking passenger flow demand and operating income as the constraint, and considering the average speed change of the bus line in the bus corridor at different times. This paper proposes a dynamic optimization model of bus route schedule based on bus Integrated Circuit Card (IC Card) data. The optimization variable is the departure frequency of the candidate lines. To solve the model, a dynamic departure interval optimization method based on improved Genetic Algorithm (GA) was designed under different decision preferences. The method includes the calibration of generalized cost functions for passengers and bus companies and grasps the characteristics of bus operating speed changes and the design of departure strategies under different decision preferences. The validity and applicability of the proposed method are verified by a numerical example. We mainly carried out the following work: (1) Dynamic analysis of the time dimension of the bus departure interval takes into account the changes in passenger time characteristics during peak periods. (2) Seven schemes of weight ratio of passenger waiting time cost and bus operation cost were designed, and the departure intervals with different benefit orientations of passengers and operators were discussed, respectively, so as to select the corresponding departure schemes for decision makers under different decision preferences. The results show that (1) the total cost of the 7 different weighting schemes is lower than the actual value by $6.90 \%$ to $18.20 \%$; (2) when decision makers need to bias the weight to the bus company, the weight ratio $\alpha$ : $\beta$ between passengers and bus company is $0.25: 0.75$ which works best. The frequency of departures has been reduced by 6 , and at the same time, the total optimized cost is reduced by $18.2 \%$; (3) when decision makers need to bias the weight to the passengers, the weight ratio $\alpha: \beta$ between the passengers and bus company is $0.75: 0.25$ which works best. The frequency of departures has been increased by 19 , and at the same time, the total optimized cost is reduced by $17.7 \%$; and (4) when decision makers consider passengers and bus companies equally, the weight ratio $\alpha: \beta$ between passengers and bus companies is $0.5: 0.5$, the optimization cost is the closest to the actual cost, the optimization cost is reduced by $6.9 \%$, and the frequency of departures has been increased by 5 . The results show that the model in this paper provides a new idea for the information mining of bus routes in the research based on the bus IC Card data and provides an effective tool for the management of different operation decision preferences.
\end{abstract}

\section{Introduction}

Improper setting of bus line schedules will cause social and economic losses and potential safety hazards. Short departure intervals will waste resources and increase the financial pressure of the bus company. Too long intervals between departures will result in longer waiting times for passengers, a waste of time for passengers, and even the accumulation of people in public places, which may cause safety accidents [1]. The bus line optimization problem includes bus line path, line length, line nonlinear coefficient, line operation timetable, vehicle capacity, and so on [2]. The research problem of this paper is the departure schedule optimization of a single bus line in the commuter corridor. Starting from the departure 
schedule, this problem optimizes the travel waiting time and bus operation cost of passengers on the whole line, so as to achieve the goal of improving passengers' riding experience and the income of bus companies [3]. Many rersearchers have conducted in-depth research on the optimization of bus timetable. At present, the research directions of bus line optimization mainly include single-line timetable optimization, cooperative optimization of multiline timetable, and considering the connection between bus and rail transit.

For single-line timetable optimization problems, Ma et al. [4] and Hassold and Ceder [5] took the passenger waiting time as the generalized cost of passengers, considered the bus operation cost and bus operation emission as the bus operation cost, and used GA to optimize the timetable. Using multisource bus data, Wen-zhou et al. [6] proposed a new time division method based on minimizing fleet operation time cost, established an optimization model with the goal of minimizing the cumulative fleet operation time cost throughout the day, and used GA to optimize the operation time division scheme. Wihartiko et al. [7], Wu et al. [8], and Tang and Yang [9] improved the GA by combining the integer programming model with the GA, setting the GA with local search and setting the quantum GA with penalty strategy to optimize the timetable for passengers and the generalized cost of bus companies. Bao-yu et al. [10] and Wang [11], respectively, designed the timetable optimization using K shortest path idea and max-min ant colony algorithm from the perspective of adding different types of buses to the operation line. Jun et al. [12], Wu et al. [13], and Zhang et al. [14] started with the service reliability of public transport companies, analyzed the suitability of bus arrival punctuality rate and passenger arrival time with the current operation timetable, and optimized the operation timetable with maximizing service reliability as a constraint. Some reasearchers considered the impact of signalized intersections in public transport operation. Among them, Jing et al. [15] considered the impact of public transport advance policy on social vehicles at signalized intersections and used simulated annealing algorithm and an event-driven policy combination model to optimize the timetable under the constraints of total line operation time and the negative impact of line operation on social vehicles at intersections. Bai et al. [16] considered the impact of the green light phase of the signal intersection on the line operation and optimized the bus schedule with the constraints of the average travel time and the average punctuality rate of the bus through the simulation software. Zhang et al. [17] and $\mathrm{Gu}$ et al. [18] proposed, from the perspective of data, the former uses the polynomial difference method to fill in the data for the lack of bus operation data and the latter uses back propagation (BP) and Radial Basis Function (RBF) neural networks to predict the boarding data of IC Card passengers and optimize the operation timetable. Yang et al. [19] proposed, from the perspective of energy conservation and emission reduction, an evaluation system for energy conservation and emission reduction when buses are driving on the road, and the bus schedule is optimized with emission pollution as the objective function.

For cooperative optimization of multiline timetable, $\mathrm{Xu}$ et al. [20] proposed a method to identify the direction of passengers getting off, transferring, and running based on the relationship between station attraction right, card swiping interval, and threshold. Zhang and Cao [21] calibrated the weight of passengers with different travel purposes, considered the demand of passengers with different travel purposes for equivalent time, and used GA to optimize the timetable. Chu et al. [22] considered the travel path selection of passengers in the area and used a mixed integer linear programming model and heuristic algorithm to optimize the operation timetable and pedestrian path selection at the same time. Jiang et al. [23], Wu et al. [24], and GuoJiang and Jian-Bo [25] used an enumeration method, GA based on nondominated sequence, and GA to optimize the operation schedule with the minimum total waiting time of passengers as the constraint. Wang and Cao [26] converted the loss of passengers caused by early and late vehicles into equivalent operating mileage. Aiming at minimizing the total operating mileage of multiple buses, considering passengers' station constraints, bus capacity constraints, and passengers' travel time window, they established a customized bus scheduling optimization model. Yang et al. [27] selected three kinds of public transit modes to establish a multimode public transit network and apply dimensionality reduction algorithm and the branch boundary method to optimize the transportation network.

Considering the connection between bus and rail transit, Takamatsu and Taguchi [28] established an event activity network to give vehicle timetable and passenger behavior in the backward areas of public transport in Japan and explored the rationality of train and bus transfer with the constraint of passenger transfer waiting time. Dou and Meng [29], based on exploring the rationality of the transfer between the terminal bus and the railway station, taking the minimization of passenger transfer waiting time as the constraint, and considering the bus capacity and passenger queuing attitude, established an MINLP model to optimize the timetable. Zheng-Wu and Ming-Qun [30] built a two-stage coordinated optimization of the operation lines of the corresponding feeder bus system at multiple transfer points for the mixed demand including reservation demand and real-time demand.

In summary, researchers at this stage are mainly concerned with the impact of the setting of bus schedules on passengers and bus companies. There are few studies on the changes in operating speed caused by the influence of social vehicles during bus operation. This article focuses on the itinerary optimization of a single line in the public transportation service corridor, extracting passenger flow characteristics from IC Card data, and grasping the characteristics of the travel time between stations on the target line on the target line based on the IC Card data of the passenger on the target line. GA is used to optimize the timetable with the travel cost model of the bus company to discuss the departure interval under different decisions.

\section{Methodology}

2.1. Problem Description. In the optimization of a single bus line, the departure interval of the bus at different times affects the bus service level, which is expressed by the total 
time cost of passengers and the operating cost of the bus company. In the research on the travel of passengers on bus lines, the travel time and travel OD matrix of passengers can be calculated according to the bus IC Card data. The research objective of this paper is to determine the dynamic departure interval of bus lines in different periods of working days. According to the obtained passenger IC Card swiping data, this paper makes the following assumptions:

(1) The running speed of the bus between bus stops is constant, which is calculated by the average running speed between two stations in different time periods

(2) There is no passenger capacity limit during the operation of the bus, which means that passengers at all stations can be loaded by the next bus currently waiting

(3) The dimensions and operating parameters of public transport vehicles are the same

Based on the abovementioned assumptions, it is determined that the main research content of this paper is the impact of passenger travel demand in different time periods and different bus speeds in different time periods on bus schedule planning.

Passenger travel demand and bus operating speed change at different times. Therefore, the research time range of this paper needs to include the peak period and peak period of passenger travel in a day, in which the passenger travel characteristics and bus operating speed are extracted through IC Card data.

The main work of this paper is shown in Table 1, problem-solving framework, which is mainly divided into three parts:

Part I: basic data acquisition: it includes obtaining necessary data such as the number of passengers arriving in the time period and the running speed of buses in different periods between stations

Part II: design and modeling of different decision preferences: the different weights between passenger waiting and bus company operating costs are designed, and the GA is used to solve the optimal cost and the corresponding departure schedule

Part III: result analysis: the optimized timetable is compared with the existing departure timetable, and the feasibility of optimizing the timetable is discussed

2.2. Model Parameter. The bus route consists of $s$ stops. According to the assumptions mentioned above, the variables and constants used in the model are as follows:

$s$ : all stops that the bus line passes through,

$s=1,2, \ldots, s-1$

$n$ : the $n$th bus, $n=1,2,3 \ldots$

$t_{s, n}$ : time when the $n$th bus arrives at the $s$ station

$t_{s, w}$ : stop time of the train at the station $a$ : starting time of the study period, in minutes

$b$ : end time of the study period, in minutes

$c$ : the time interval set in the study, in minutes

$T_{x}$ : average running time of the bus in time period $x$, $x=1,2,3 \ldots c / b-a$

$q_{t}$ : number of passengers arriving at the station per minute during the period from $t_{s, n}$ to $t_{s, n-1}$

$\alpha$ : passenger time cost weight

$F_{W}$ : total waiting time cost of passengers

$\beta$ : weight of operating cost per bus

$F_{b}$ : total operating costs of bus companies

2.3. Generalized Cost Model Based on Decision Preferences. In this paper, the total cost model of bus line operation is composed of the waiting time cost of passengers and the operating cost of bus companies. Considering that in practical problems, decision makers often have different preferences for the interests of passengers and bus companies, that is, different decision preferences. Therefore, in this paper, different weights $(\alpha, \beta)$ are set for passenger waiting time and bus company operating expenses. The impact of different preferences of decision makers on the total cost of bus line operation is discussed.

2.3.1. Time Cost of Passenger Waiting. In the waiting time cost of passengers, it is defined that the arrival time of passengers obeys Poisson distribution in the corresponding departure interval, and the total waiting time cost of passengers can be expressed as follows:

$$
F_{w}=\left[\sum_{s=1}^{S} \sum_{n=1}^{N} \frac{1}{2}\left(t_{s, n}-t_{s, n-1}\right) \sum_{t=t_{s, n-1}}^{T=t_{s, n}} q_{t}\right] \alpha .
$$

2.3.2. Operating Costs of Public Transport Companies. In the operating costs of public transport companies, it is defined that the operating costs of public transport companies are related to the driving time of each bus on the road. The longer the driving time, the higher the operating costs of public transport companies. Through the analysis of the travel time of each stop, it is obtained that the travel characteristics of bus stops comply with the density function characteristics of lognormal distribution. The operation cost model of bus companies is shown as follows:

$$
F_{b}=\beta \int_{t_{s, n}+t_{s, w}}^{t_{s+1, n}} \frac{\sum_{1}^{(c / b-a)} T_{X}}{t \delta \sqrt{2 \pi}} e^{-\left((\ln t-\mu) / 2 \delta^{2}\right)}+T_{1} .
$$

2.3.3. Generalized Cost Model Based on Decision Preference. The model is built with the goal of minimizing the sum of passenger waiting time cost and bus company operating cost under different weights. The model is shown as follows: 
TABle 1: Problem-solving framework.

\begin{tabular}{|c|c|}
\hline Steps & Main work \\
\hline Eliminating useless data of IC Card & Data preprocessing \\
\hline Determining peak and off-peak periods & $\begin{array}{l}\text { Determining the optimization } \\
\text { period }\end{array}$ \\
\hline $\begin{array}{l}\text { Extracting card swiping time data and analyzing passenger flow characteristics of card swiping time data } \\
\text { and bus running speed }\end{array}$ & Key factor analysis \\
\hline $\begin{array}{l}\text { Weighting design under different decision preferences of optimization of departure schedule by GA } \\
\text { Comparison between the optimized and original departure timetable }\end{array}$ & $\begin{array}{l}\text { Model solving } \\
\text { Analysis }\end{array}$ \\
\hline
\end{tabular}

$$
\begin{aligned}
& \operatorname{Min} M=F_{w}+F_{b}, \\
& \quad(1) w_{\min } \leq t_{s, n}-t_{s, n-1} \leq w_{\max }, \\
& \text { s.t., } \quad \text { (2) } t_{s, n} \in 1,2,3 \ldots
\end{aligned}
$$

\section{Model Framework}

3.1. Algorithm Flow Chart. GA is a biological evolution calculation model simulating Darwin's biological evolution theory. It uses computer simulation to transform the problemsolving process into a process similar to the crossover and mutation of chromosome genes in biological evolution.

In the optimization problem of public transportation schedule, the interval running time variable has discrete characteristics, which is a typical nondeterministic polynomial (NP-hard) problem. The self-adaptability and global search of genetic algorithm have good performance in solving NP problems. In addition, the form of chromosome cross mutation fits the bus departure timetable. This paper finally chooses improved GA as the model for optimization.. The flow chart of the algorithm is shown in Figure 1.

3.2. Encoding. Encoding is the focus of the model solving process. In order to realize the dynamic optimization of bus timetable, each gene in a chromosome is defined as a time point. The encoding length is determined by the optimization time period. If the study period is 7:00-8:30, and the unit time is set as $1 \mathrm{~min}$, and the number of genes in a chromosome is 90 . The gene expression form is $0-1$, where 0 means no departure at the corresponding time point and 1 means departure at the corresponding time point. It is also stipulated that the unit departure interval of the bus is $1 \mathrm{~min}$. Because of the randomness of the number of arrivals, the same phenotype of different gene chains can be avoided. The final chromosome structure is shown in Figure 2.

3.3. Initialize Population. The initialization population is used to generate realistic random chromosomes. For the population initialization of bus operation timetable, we mainly focus on the rationality of departure interval. It is necessary to eliminate randomly generated chromosomes with unreasonable departure interval.

3.4. Fitness Function. The function of fitness function is to evaluate and distinguish the advantages and disadvantages of population individuals. In this paper, the objective function to be solved is taken as the fitness function. Therefore, the fitness function corresponding to the minimization of the objective function in the bus timetable optimization is shown as follows:

$$
f_{D}=\min \left(f_{1}+f_{2}\right) \text {. }
$$

\section{Analysis and Discussion}

4.1. Bus Line Information. No. 647 bus is one of the bus lines in the commuter corridor between Central Business District (CBD) and Tongzhou District. The main purpose of travel is work travel. Through IC Card data, it can be found that travel behavior has obvious time characteristics. The number of passengers in different periods fluctuates greatly, which is suitable for discussion according to the dynamic passenger flow demand. Therefore, this paper discusses and analyzes the No. 647 bus line. The basic operation data of bus No. 647 are shown in Table 2.

4.2. IC Card Data Structure. Data include several features, such as bus time, transaction time, travel route number, onand-off station number, and IC Card number, Table 3.

4.3. Line Passenger Flow. The uplink data of the No. 647 bus is optimized. Then, the boarding time of passengers and the number of arrivals per minute during the study period are analyzed. Finally, the frequency distribution diagram is shown in Figure 3.

According to the distribution map of passenger flow arrival, the passenger flow characteristics of No. 647 bus uplink show a single peak distribution in a single day operation cycle.

The passenger flow of 2 hours in the morning peak from 6:30 to $8: 30$ am accounts for $32 \%$ of the passenger flow of the whole day. The number of passenger flow reachs 786 persons/hour in peak hour, and it is 207 persons/hour in offpeak. The passenger flow dropped rapidly after 7 pm. 5:30 am to $10: 30$ am is selected as the analysis period shown in Figure 4.

4.4. Impact of Average Operation Speed between Stations. If the road operating conditions in different periods of time is different, the times required for buses are also different. The longer the driving time is, the more the maintenance and other expenses the bus company spends. This paper addresses the impact of the average bus 


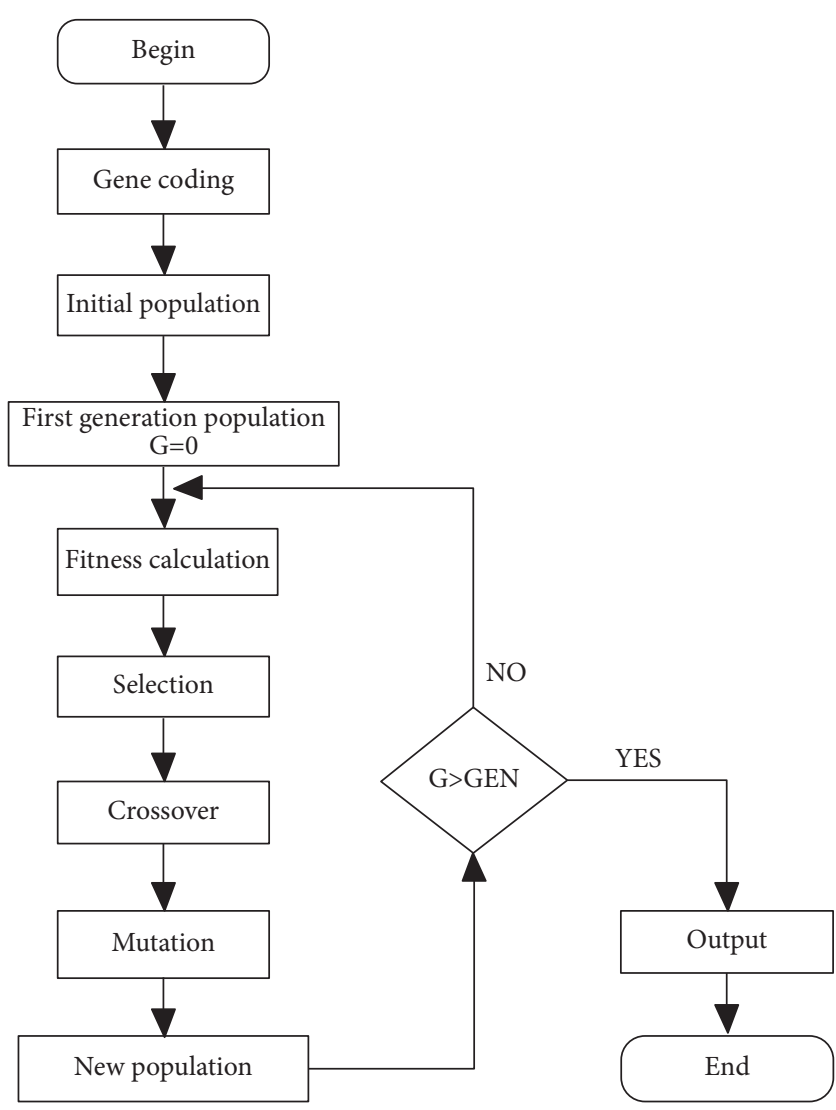

FIGURE 1: Flow chart of the algorithm.

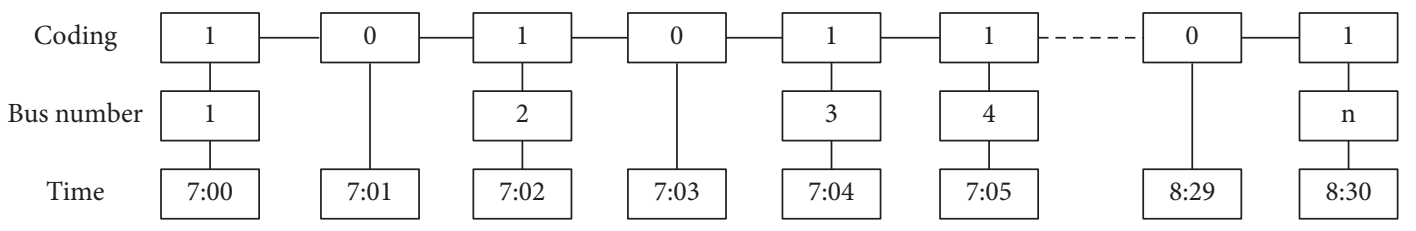

FIgURE 2: Structure of chromosome.

TABle 2: Basic operation data of the No. 647 bus.

Line name

Number of sites

Number of sites

Number of operating vehicles (vehicles)

Number of guests (person)

Operating time (upside)

TABle 3: Information structure of bus IC Card.

\begin{tabular}{lc}
\hline Attribute & Information \\
\hline Boarding time & 20180305114800 \\
Trading time & 20180305115712 \\
No. of lines & 647 \\
Boarding station & 9 \\
Drop off station & 6 \\
Card number & $1000^{* * * * * * * *} 0870$ \\
\hline
\end{tabular}




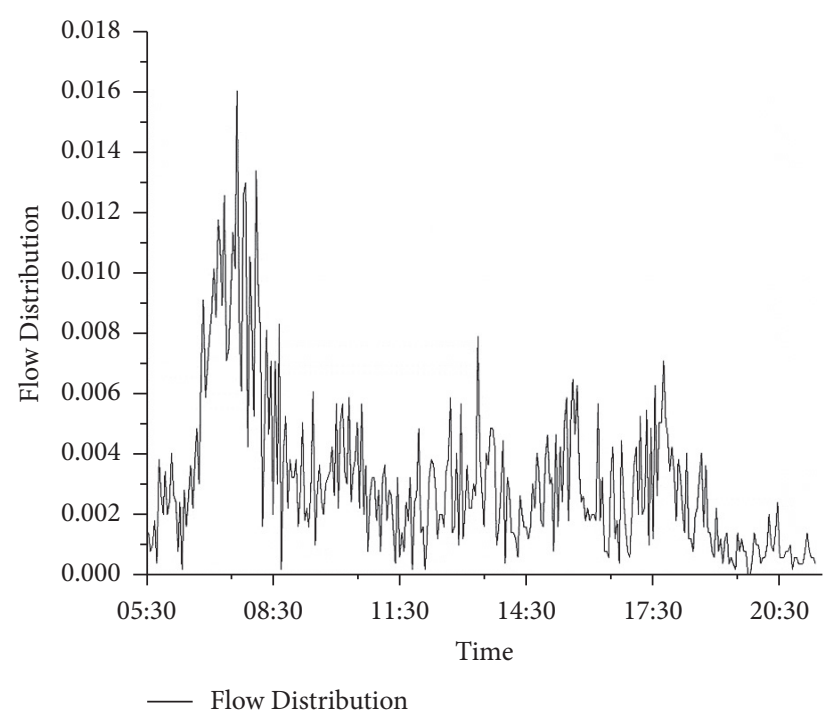

FIGURE 3: Distribution of card swiping frequency of uplink passengers.

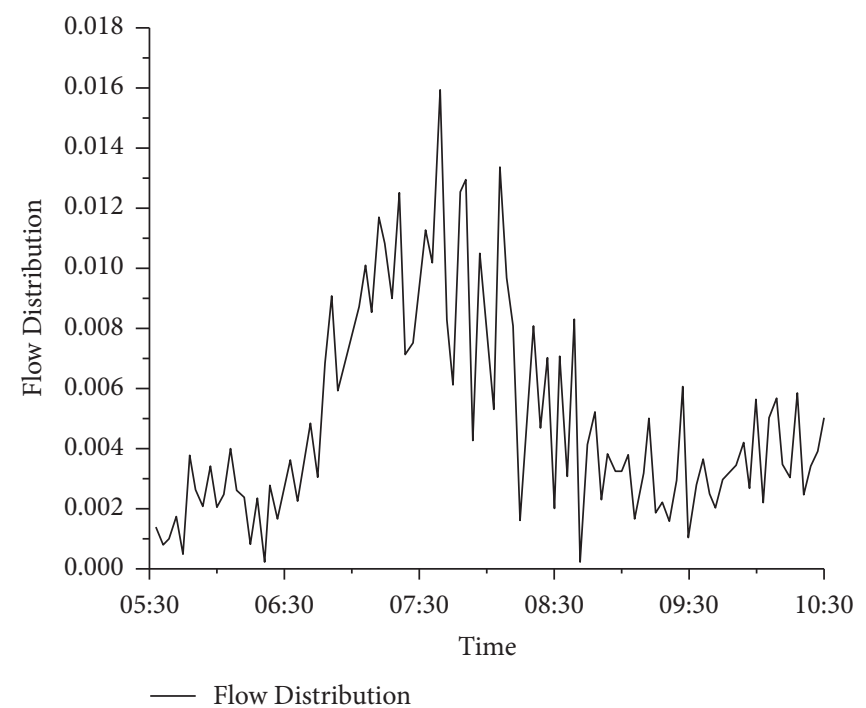

Figure 4: Frequency distribution of card swiping from 5:30am to 10:30 am.

running time between stations. Analysis was performed by on-and-off time of passengers in the IC Card data. The travel time between bus stops is extracted from the last passenger's card swiping behavior at station $s$ to the first passenger's card swiping behavior at station $s+1$. The card swiping behavior also includes card swiping when getting on and off. The total travel time of the line is divided into 15 periods. The interval is $20 \mathrm{~min}$ in the period of 5:30 am-10:30 am. By observing the travel time in different periods, it is found that the variation of travel time follows the characteristics of lognormal distribution. Lognormal distribution fitting is carried out for the travel time of the line in different periods. The travel time fitting diagram in Figure 5 and the total travel time fitting parameters are shown in Table 4. The travel time between stations is shown in Table 5.
4.5. Model Optimization Results. According to the actual departure interval of the line obtained from IC Card data mining, the minimum departure interval is specified as $\varpi=1 \mathrm{~min}$, and the maximum departure interval is $\mathrm{min}_{\max }$. The setting of departure interval is an integral multiple of $1 \mathrm{~min}$.

The parameters of the GA in the Matlab program are shown in Table 6. The actual departure of the line schedule during the period of 5:30-10:30 is shown in Table 7 .

$\alpha, \beta$ represent the coefficient of passenger waiting fee and bus company operating cost, respectively. The actual decision makers with different decision preferences may pay different attention to passengers and bus companies in designing the bus schedule. Cost and expense comparisons at the different weight ratios are shown in Table 8. 


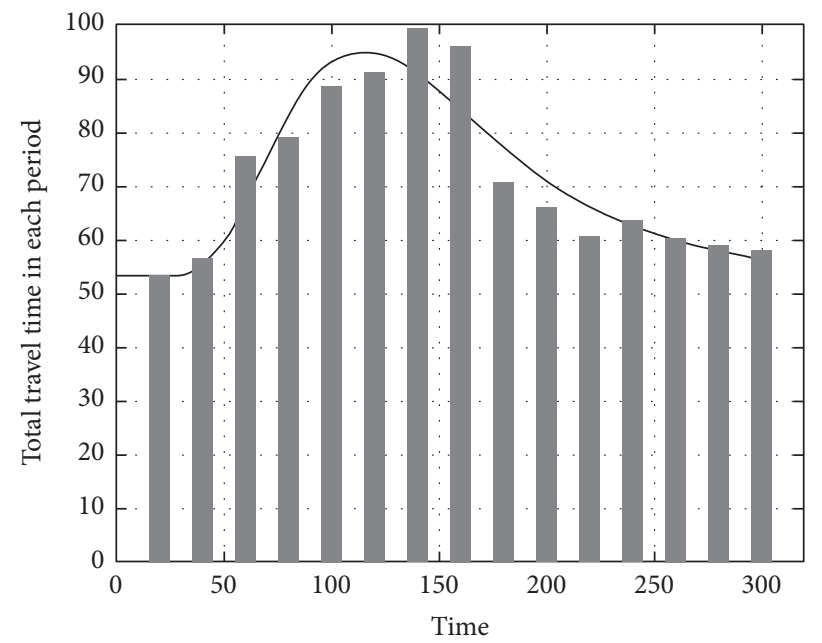

FIgURE 5: Line travel time fit diagram for the study period.

TABLE 4: Line travel time fitting parameters during the study period.

\begin{tabular}{lcc}
\hline Total full site runtime $(\min )$ & $\mu$ & $\Delta$ \\
\hline 1075.64 & 4.939 & 0.406 \\
\hline
\end{tabular}

TABLe 5: Travel time fitting parameters between the stations during the study period.

\begin{tabular}{|c|c|c|c|}
\hline Site number & Total running time in the whole period $(\mathrm{min})$ & $\mu$ & $\delta$ \\
\hline $1-2$ & 49.23 & 5.314 & 0.660 \\
\hline $2-3$ & 43.92 & 5.591 & 0.675 \\
\hline $3-4$ & 31.55 & 4.430 & 0.427 \\
\hline $4-5$ & 35.68 & 4.871 & 0.672 \\
\hline $5-6$ & 29.92 & 4.943 & 0.457 \\
\hline $6-7$ & 28.13 & 4.955 & 0.744 \\
\hline $7-8$ & 46.81 & 5.112 & 0.585 \\
\hline $8-9$ & 80.43 & 4.992 & 0.559 \\
\hline $9-10$ & 58.42 & 4.953 & 0.618 \\
\hline $10-11$ & 40.01 & 4.648 & 0.635 \\
\hline $11-12$ & 43.22 & 4.828 & 0.218 \\
\hline $12-13$ & 59.06 & 4.906 & 0.118 \\
\hline $13-14$ & 64.20 & 5.309 & 0.637 \\
\hline $14-15$ & 71.30 & 5.772 & 1.046 \\
\hline $15-16$ & 97.48 & 4.989 & 0.270 \\
\hline $16-17$ & 276.97 & 4.748 & 0.184 \\
\hline $17-18$ & 39.31 & 4.814 & 0.189 \\
\hline
\end{tabular}

Taking $\alpha: \beta=0.33$ : 0.66 as an example, the number of departures is 31 and close to 33 the actual number of departures. The running results of GA are shown in Figure 6. Before the 175th generation of iteration, the optimal individual target value shows a downward trend. Therefore, the optimal solution is reached after the 175th generation, and the departure time of line 5:30-10:30 is obtained, as shown in Table 9.

4.6. Discussion. This paper focuses on the dynamic optimization of the timetable of a major bus line in the commuter bus corridor. Considering the comprehensive benefits of passengers and bus companies, the improved GA is used to optimize the dynamic timetable. The cost ratio between passenger waiting and bus operation is divided into seven kinds. When the ratio of $\alpha$ : $\beta$ is $0: 1$ or $1: 0$, it is considered that the two strategies are extreme and will not abandon the interests of one party in actual operation. In this paper, they are only used as algorithm examples in different cases.

4.6.1. Decision Making Based on Comprehensive Cost Considerations. The total costs of the seven schemes are less than the current departure schedule (shown in Tables 7 and 8 ). When the ratio $\alpha$ : $\beta=0.25: 0.75$, best effect is gotton. The total cost is $81.8 \%$ of the actual cost. When $\alpha: \beta=0.5: 0.5$, the total cost is the closest to the actual cost, which is $93.1 \%$ 
TABLE 6: Driving time fitting parameters between the stations during the study period.

\begin{tabular}{lccc}
\hline Population quantity & Iterations & Crossover probability & Variation probability \\
\hline 100 & 800 & 0.8 & 0.025 \\
\hline
\end{tabular}

TABle 7: Actual line schedule.

\begin{tabular}{lcccc}
\hline Minimum departure interval $(\mathrm{min})$ & Maximum departure interval $(\mathrm{min})$ & Number of vehicles & Total cost value \\
\hline 3 & 23 & 33 & 4640 \\
\hline
\end{tabular}

TABLe 8: Comparison of total cost values under different decisions.

\begin{tabular}{lcccc}
\hline$\alpha: \beta$ & Number of vehicles & Total cost value & Coparision with actual cost & Cost reduction ratio \\
\hline $0: 1$ & 11 & 1427 & $30.7 \%$ & $69.30 \%$ \\
$0.25: 0.75$ & 25 & 3799 & $81.8 \%$ & $18.20 \%$ \\
$0.33: 0.66$ & 31 & 4102 & $88.4 \%$ & $11.60 \%$ \\
$0.5: 0.5$ & 38 & 4319 & $93.1 \%$ & $6.90 \%$ \\
$0.66: 0.33$ & 42 & 4037 & $87 \%$ & $13.00 \%$ \\
$0.75: 0.25$ & 52 & 3817 & $82.3 \%$ & $17.70 \%$ \\
$1: 0$ & 301 & 125 & $2.7 \%$ & $97.30 \%$ \\
\hline
\end{tabular}

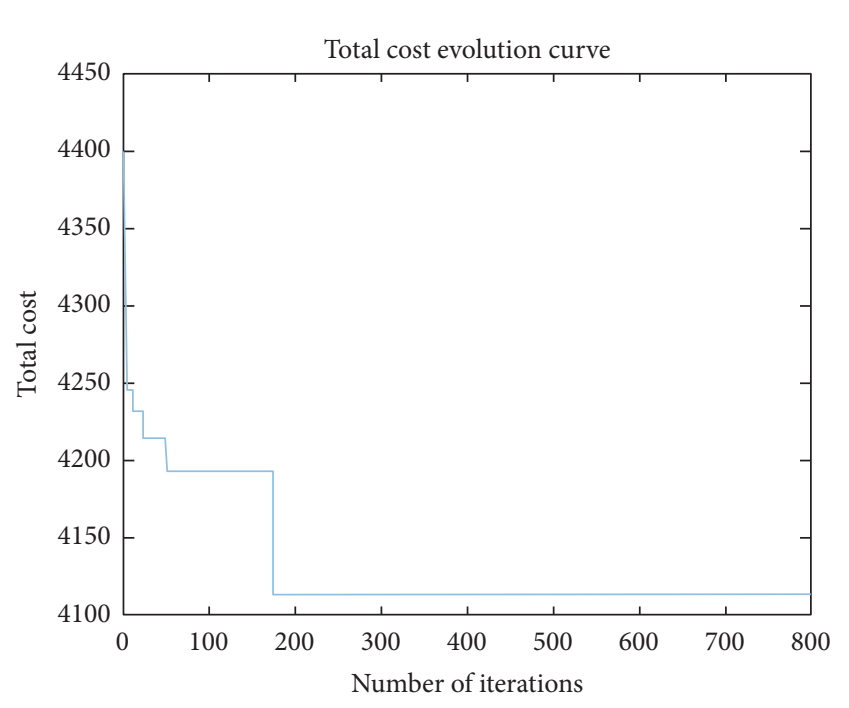

FIgURE 6: Iterative process of optimal solution.

of the actual cost. Also when the ratio is $\alpha: \beta=0.33: 0.66$, the departure frequency is similar to the actual situation, and the total general operating cost of 4201 is reduced by $12 \%$ from the actual general operating cost of 4640 . The model meets the optimization of the characteristics of actual passenger flow and multiperiod travel time under all designed weight ratios. It is proved that the total operation cost of passengers and bus companies can be further reduced in the design of departure frequency under the consideration of the actual bus holding of the bus company, which reflects the practical application value of the model in this paper.

4.6.2. Decision Making Based on the Passenger Interest Perspective. When the ratio is $\alpha: \beta=0.66: 0.33$ or $\alpha: \beta=0.75: 0.25$, the the passenger interests is selected as a dominant factor. In order to reduce the waiting time of passengers, bus companies need to improve the departure frequency and provide more bus vehicles to meet the travel needs. Then, compared with the actual departure situation, the number of public transport vehicles required is $27.3 \%$ and $57.6 \%$ more. It can be found that as the interests are gradually biased towards passengers, the growth rate of departure times has become larger. The number of buses in the bus company's stations is limited. Excessive consideration of passengers' interests may increase the pressure on the number of vehicles held by the bus company when ensuring the overall cost reduction.

4.6.3. Decision Making Based on the Interests of Public Transport Companies. When the ratio is $\alpha: \beta=0.25: 0.75$ and $\alpha: \beta=0.33: 0.66$, the decision makers mainly focus on the operating interests of the bus company. In this case, the departure frequency increases significantly. When the ratio is $0.25: 0.75,25$ departures are expected. In this case, the number of departures is more than twice that when the ratio is $0: 1$. When the ratio is $0.33: 0.66,31$ departures are expected and 6 times more than $\alpha$ : $\beta=0.25: 0.75$. It can be seen that as the interest to the bus company gradually increases, the reduction rate of the number of deparincreases. In this process, the reduction of departure number will lead to the increase of departure interval, which will directly lead to the increase of waiting time of arriving passengers and reduce passengers satisfaction.

In summary, different focus targets lead to different revenue and departure times. The greater the ratio of $\alpha: \beta$ is, the greater the tendency to consider the waiting time of passengers. When it is 0.5:0.5, a better balance is obtained. When decision makers want to take into account the interests of passengers and bus companies at the same time, the total generalized cost may become larger when the existing passenger flow characteristics remain unchanged. It is considered that the line satisfaction survey can be conducted for line passengers in time, and the departure times and 
TABLE 9: Optimised line departure schedule.

\begin{tabular}{lcccccccc}
\hline Train number & 1 & 2 & 3 & 4 & 5 & 6 & 7 & 8 \\
\hline Time & $5: 30$ & $5: 37$ & $5: 53$ & $5: 58$ & $6: 10$ & $6: 13$ & $6: 24$ & $6: 28$ \\
Train number & $\mathbf{9}$ & $\mathbf{1 0}$ & $\mathbf{1 1}$ & $\mathbf{1 2}$ & $\mathbf{1 3}$ & $\mathbf{1 4}$ & $\mathbf{1 5}$ & $\mathbf{1 6}$ \\
Time & $6: 36$ & $6: 44$ & $6: 54$ & $7: 02$ & $7: 07$ & $7: 14$ & $7: 21$ & $7: 23$ \\
Train number & $\mathbf{1 7}$ & $\mathbf{1 8}$ & $\mathbf{1 9}$ & $\mathbf{2 0}$ & $\mathbf{2 1}$ & $\mathbf{2 2}$ & $\mathbf{2 3}$ & $\mathbf{2 4}$ \\
Time & $7: 28$ & $7: 33$ & $7: 37$ & $7: 51$ & $8: 10$ & $8: 32$ & $8: 48$ & $9: 00$ \\
Train number & $\mathbf{2 5}$ & $\mathbf{2 6}$ & $\mathbf{2 7}$ & $\mathbf{2 8}$ & $\mathbf{2 9}$ & $\mathbf{3 0}$ & $\mathbf{3 1}$ & \\
Time & $9: 12$ & $9: 30$ & $9: 33$ & $9: 45$ & $10: 01$ & $10: 22$ & $10: 30$ & \\
\hline
\end{tabular}

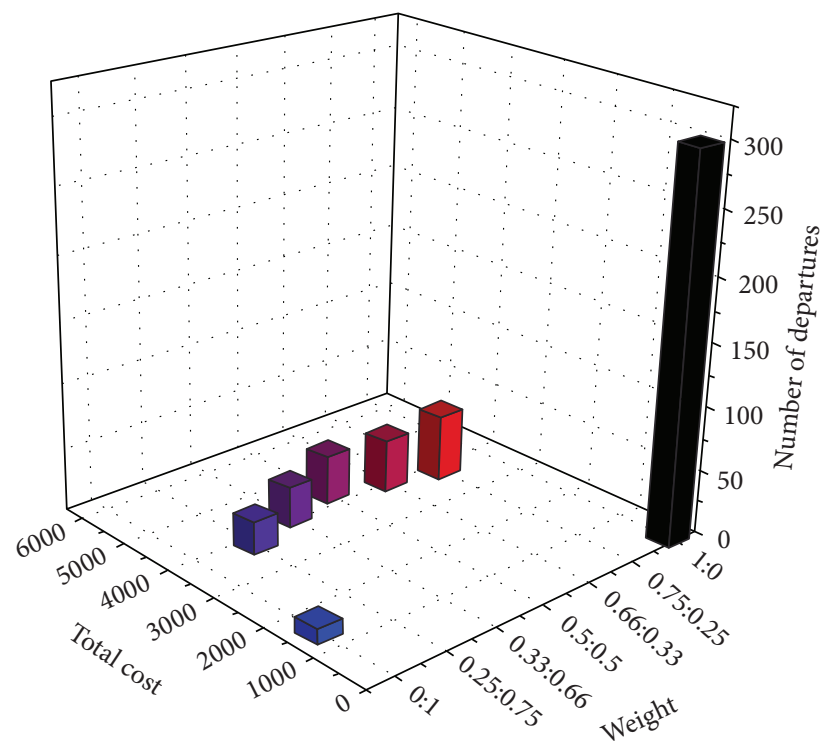

Figure 7: Total cost and the number of departures under different weights.

corresponding departure intervals can be adjusted within the range of ensuring passenger satisfaction and the number of buses in the bus yard and station can bear the departure times, so as to obtain a lower total generalized cost value and improve the social value of the line shown in Figure 7.

\section{Conclusions}

Based on the arrival characteristics of passenger flow and bus travel time between stations in different time periods, taking into account the operating costs of bus companies and passenger waiting time costs, this paper constructs the schedule optimization model of urban bus lines under dynamic demand and studies the operation strategies under different better decisions, which has certain guiding significance for bus line operation. However, there is still some room for improvement in this paper. In this paper, in order to take into account the impact of the full load rate in the line operation on the passenger riding experience and the impact of multiline transfer in the bus network, further improvement is needed in the follow-up research.

The main conclusions of this paper are as follows.

(1) The passenger travel characteristics and waiting time distribution on the bus corridor are analyzed.
(2) A dynamic optimization model of bus timetable in commuter corridor is proposed, which comprehensively considers the interests of passengers and operating companies.

(3) A model solving method based on improved GA is constructed. The income values under different weights are compared and analyzed.

\section{Data Availability}

The data used to support the findings in this study are available from the corresponding authors upon request.

\section{Ethical Approval}

All methods were carried out in accordance with relevant guidelines and regulations. All experimental protocols were approved by a named institutional and/or licensing committee.

\section{Consent}

Informed consent was obtained from all subjects and/or their legal guardian (s).

\section{Conflicts of Interest}

The authors declare no conflicts of interest.

\section{Acknowledgments}

This work was supported by the National Social Science Foundation (21FGLB014).

\section{References}

[1] Z. Li, Y. Dong, Y. Wen, H. Xu, and J. Wu, “A deep pedestrian tracking SSD-based model in the sudden emergency or violent environment," Journal of Advanced Transportation, vol. 2021, Article ID 2085876, 13 pages, 2021.

[2] C. W. Ya, L. X. Fei, and W. L. Jiang, "Data-driven optimization methods for dynamic transit network adjustment," Journal of Transportation Systems Engineering and Information Technology, vol. 17, no. 6, pp. 114-119, 2017.

[3] R. Song, S. He, Y. Yang, H. Yang, and K. Luo, "Integrated optimization model of transit scheduling plan and bus use," Journal of Traffic and Transportation Engineering, vol. 19, no. 3, pp. 70-76, 2006.

[4] X. L. Ma, X. L. Shen, Z. Zhao, L. Sen, and C. Xi, "Optimization of autonomous bus scheduling based on Lagrangian relaxation," China Journal of Highway and Transport, vol. 32, no. 12, pp. 10-24, 2019.

[5] S. Hassold and A. Ceder, "Multiobjective approach to creating bus timetables with multiple vehicle types," Transportation Research Record, vol. 2276, no. 1, 2012.

[6] J. I. N. Wen-zhou, L. I. Peng, and W. U. Wei-tiao, "Time-ofday interval partition method for bus schedule based on multi-source data and fleet-time cost optimization," China Journal of Highway and Transport, vol. 32, no. 2, pp. 143-154, 2019.

[7] F. D. Wihartiko, A. Buono, and B. P. Silalahi, "Integer programming model for optimizing bus timetable using GA," 
IOP Conference Series: Materials Science and Engineering, vol. 166, no. 1, 2017.

[8] Y. Wu, J. Tang, Y. Yang, and Z. Pan, "A stochastic optimization model for transit network timetable design to mitigate the randomness of traveling time by adding slack time," Transportation Research Part C Emerging Technologies, vol. 52, 2015.

[9] X. L. Tang and S. H. Yang, "A improved bus timetable scheduling model using quantum GA based on penalty strategy," Applied Mechanics and Materials, vol. 253-255, 2013.

[10] H. Bao-yu and Y. Pang, P. Y. long, Multi-type bus timetable optimization considering unbalanced passenger flow in time and space," Journal of South China University of Technology, vol. 48, no. 11, pp. 38-48, 2020.

[11] Y. Wang and J. Q. Shen, "Single line transit mixed scheduling model based on vehicle departure timetable," Computer Science, vol. 44, no. 10, pp. 269-275, 2017.

[12] L. I. Jun, Y. X. Deng, and L. H. Huang, “Arrangement and evaluation of bus arrival timetable based on service reliability," Acta Scientiarum Naturalium Universitatis Sunyatseni, vol. 59, no. 5, pp. 86-94, 2020.

[13] Y. Wu, H. Yang, J. Tang, and Y. Yang, "Multi-objective resynchronizing of bus timetable: model, complexity and solution," Transportation Research Part C, vol. 67, 2016.

[14] M. J. Zhang, C. Chen, and M. X. Han, "Passenger waiting time and behavioral adaption to suburban bus timetable," Applied Mechanics and Materials, vol. 505-506, 2014.

[15] Y. Jing, S. J. gang, and W. T. Jian, "Optimizing tram timetables considering the negative effects at intersections," Systems Engineering-Theory \& Practice, vol. 39, no. 10, pp. 2676-2688, 2019.

[16] H. J. Bai, G. X. Ren, and R. J. Dong, "Transit signal priority method based on schedule under connected vehicle environment," Journal of Chongqing Jianzhu University, vol. 37, no. 7, pp. 85-91, 2018.

[17] K. Zhang, Z. Y. Qi, and S. Jie, "Arrival time filling method based on bus operation data," Shandong Science, vol. 33, no. 4, pp. 83-90+116, 2020.

[18] J. J. Gu and Z. B. Jiang, "An optimization of bus timetable during peak periods based on forecasts passenger flow using neural network," Journal of Transport Information and Safety, vol. 35, no. 2, pp. 109-114, 2017.

[19] Y. Yang, Z. Yuan, J. Chen, and M. Guo, "Assessment of osculating value method based on entropy weight to transportation energy conservation and emission reduction," Environmental Engineering and Management Journal, vol. 16, no. 10, pp. 2413-2423, 2017.

[20] W. Y. Xu, C. Y. Deng, and B. Y. Liu, "Approach on public traffic passenger flow statistics based on IC data," Journal of Transport Information and Safety, vol. 26, no. 6, pp. 33-39+95, 2015.

[21] Y. Zhang and Z. Y. Cao, "Optimization model for public transport timetable with the time weight of transfer station," Journal of Transportation Engineering and Information, vol. 18, no. 1, pp. 77-82+98, 2020.

[22] J. C. Chu, K. Korsesthakarn, Y. T. Hsu, and H. Y. Wu, "Models and a solution algorithm for planning transfer synchronization of bus timetables," Transportation Research Part E, vol. 131, 2019.

[23] X. H. Jiang, G. Xiu-Cheng, and X. 1. Gong, "Integrated optimization for timetabling and vehicle scheduling of zone urban and rural bus," Journal of Transportation Systems
Engineering and Information Technology, vol. 19, no. 3, pp. 141-148, 2019.

[24] W. U. Ying-Hui, Z. H. U. Yi-Fan, and J. I. Ying, "A multiobjective optimization model for Re-synchronizing of bus network timetable," Operations Research and Management Science, vol. 26, no. 11, pp. 42-48+64, 2017.

[25] S. Guo-Jiang and D. Jian-Bo, "Research on two-line bus transferring model based on non-equal intervals," Journal of Zhejiang University of Technology, vol. 45, no. 5, pp. 587-590, 2017.

[26] J. Wang and Y. Cao, "Customized bus route vehicle schedule method considering travel time windows," China Journal of Highway and Transport, vol. 31, no. 5, pp. 143-150, 2018.

[27] Y. Yang, Z. Yuan, J. Li, and Y. Wang, "Multi-mode public transit OD prediction and scheduling model," Advances in Transportation Studies, vol. 3, pp. 133-146, 2018.

[28] M. Takamatsu and A. Taguchi, "Bus timetable design to ensure smooth transfers in areas with low-frequency public transportation services," Transportation Science, vol. 54, no. 5, 2020.

[29] X. Dou and Q. Meng, "Feeder bus timetable design and vehicle size setting in peak hour demand conditions," Transportation Research Record, vol. 2673, no. 10, 2019.

[30] W. Zheng-Wu and S. Ming-Qun, "Coordinated optimization of operation routes for responsive feeder transit systems with multiple transfer points," China Journal of Highway and Transport, vol. 32, no. 9, pp. 164-174, 2019. 